\title{
Toxoplasmosis ocular: frecuencia y características clínicas en un consultorio de oftalmología pediátrica
}

\section{Ocular Toxoplasmosis: frequency and clinical characteristics in a pediatric ophthalmology clinic}

\author{
Olivia Cardozo ${ }^{1}$, Mirta Mesquita $^{1}$, Laura Godoy ${ }^{1}$
}

\section{RESUMEN}

Introducción: La toxoplasmosis afecta frecuentemente la cámara posterior del ojo y puede producir pérdida de la visión. Objetivo: Describir la frecuencia y características clínicas de la Toxoplasmosis ocular en un consultorio de oftalmología pediátrica. Materiales y Métodos: Estudio observacional, descriptivo retrospectivo. Se analizaron las historias clínicas de pacientes de 0 a 18 años de edad, con toxoplasmosis ocular, atendidos en el consultorio de oftalmología pediátrica, desde enero 2011 a mayo de 2014. Variables: edad, sexo, forma de toxoplasmosis, tipo de lesión ocular, localización de la lesión, compromiso unilateral o bilateral, y complicaciones oculares. Los datos se analizaron con SPSSv21 utilizando estadísticas descriptivas e inferenciales. No fue necesario el consentimiento informado porque se trata de un estudio retrospectivo. Resultados: Ingresaron 72 pacientes. La mediana de edad 48 meses, fueron neonatos el 6,9\%. El $52,8 \%$ eran sexo femenino. El $80,5 \%$ presentaron toxoplasmosis adquirida y el $19,4 \%$ congénita. Todos los pacientes tenían coriorretinitis. Y en el $7 \%$ se acompañaron de uveítis. La afectación fue bilateral en $52.8 \%$. Las lesiones eran inactivas en $87,5 \%$ con localización central. Presentaron complicaciones en el 58,3\% de los pacientes: estrabismo en $76 \%$ y en $24 \%$ desprendimiento de retina. Conclusión: La frecuencia de TO fue del 0,8\%. La mayoría de los pacientes tenían más de 2 años de edad y el 19\% tenía toxoplasmosis congénita. Todos tuvieron corioretinitis, con compromiso bilateral y localización central más frecuentemente. Hubo predominio de lesiones inactivas tanto en la toxoplasmosis congénita como en la adquirida. Las complicaciones fueron estrabismo y desprendimiento de retina.

Palabras claves: Toxoplamosis ocular, niños, coriorretinitis, complicaciones, uveitis.

\begin{abstract}
Introduction: Toxoplasmosis frequently affects the posterior chamber of the eye and can cause vision loss. Objective: To describe the frequency and clinical characteristics of ocular toxoplasmosis in a pediatric ophthalmology clinic. Materials and Methods: this was an observational, descriptive and retrospective study. We reviewed the clinical histories of patients from 0 to 18 years of age, with ocular toxoplasmosis, treated in the pediatric ophthalmology clinic, from January 2011 to May 2014. Variables were age, sex, form of toxoplasmosis, ocular lesion type, lesion location, unilateral or bilateral involvement, and ocular complications. The data was analyzed with SPSSv21 using descriptive and inferential statistics. Informed consent was not necessary because it was a retrospective study. Results: 72 patients were included. The median age was 48 months; $6.9 \%$ were neonates, $52.8 \%$ were female. $80.5 \%$ had acquired toxoplasmosis and $19.4 \%$ congenital. All the patients had chorioretinitis and $7 \%$ also had uveitis. Bilateral affectation was present in $52.8 \%$. The lesions were inactive at $87.5 \%$ with central location. Complications were present in $58.3 \%$ of patients: strabismus in $76 \%$ and $24 \%$ had retinal detachment. Conclusion: The frequency of OT was $0.8 \%$. The majority of the patients were over 2 years of age and $19 \%$ had congenital toxoplasmosis. All had chorioretinitis, with bilateral involvement and central location present more frequently. There was a predominance of inactive lesions in both congenital and acquired toxoplasmosis. The most common complications were strabismus and retinal detachment.
\end{abstract}

Keywords: Ocular toxoplasmosis, children, chorioretinitis, complications, uveitis.

\footnotetext{
${ }^{1}$ Hospital General Pediátrico "Niños de Acosta Nu". San Lorenzo, Paraguay.

Correspondencia: Mirta Mesquita Correo: mirtanmr@gmail.com

Conflicto de interés: Los autores declaran no poseer conflicto de interés

Recibido: 21/10/2018; Aceptado: 28/12/2018

DOI: https://doi.org/10.31698/ped.45032018006
} 


\section{INTRODUCCIÓN}

El Toxoplasma gondii, es un protozoario intracelular, adquirido frecuentemente a través del consumo de verduras, carnes no bien cocidas y por contacto con heces de felinos contaminados con ooquistes. Es menos frecuente la transmisión por vía placentaria, materno fetal, que produce el cuadro de toxoplasmosis congénita. Se estima que aproximadamente un tercio de la población mundial se ha infectado por este patógeno. En Latinoamérica la seroprevalencia varía de un país a otro desde 21 al $76 \%{ }^{(1)}$.

La mayoría de los pacientes tanto adultos como niños son asintomáticos, exceptuando los casos graves de toxoplasmosis congénita y las personas inmuno comprometidas. Este patógeno se disemina por todo el organismo y afecta principalmente el sistema nervioso central, los ojos, músculos y placenta $^{(2)}$.

La toxoplasmosis es la causa más frecuente de uveítis posterior. Típicamente afecta la cámara posterior del ojo, con lesiones únicas o múltiples. Se presenta tanto en la infección congénita como en la adquirida $^{(3)}$.

Desde hace mas de 2 décadas se conocen 15 genotipos diferentes de $T$ Gondii, la mayoría de los cuales se clasifican dentro de 1 de los 3 linajes genéticos denominados Tipo I, II y III. Dentro de estos tipos se han descubierto varios halogrupos o series de alelos ubicados en los cromosomas de los parásitos. Estos se distribuyen en diferentes áreas geográficas y en huéspedes con diferente estado inmunitario. Esta compleja estructura genética del parásito se relaciona con las diversas sero prevalencias encontradas en diferentes regiones y países. Existen pocos estudios con datos ciertos sobre la prevalencia de la toxoplasmosis ocular (TO). Podría ser más prevalente en Sudamérica en relación a otros continentes ${ }^{(4)}$. En Brasil, examinando los especímenes del banco de ojos, utilizando la reacción en cadena de la polimerasa (PCR DNA) se han encontrado de $T$ gondii en el $87 \%$ de los ojos estudiados en una región del sur del país ${ }^{(5)}$.

En Paraguay no hay programas de screening de toxoplasmosis durante el embarazo así como tampoco hay estadísticas de los niños con toxoplasmosis. Hasta donde se investigo no hay publicaciones en el país de TO en adultos o niños. El objetivo del presente trabajo es conocer la frecuencia y las características de la TO en un consultorio de oftalmología de un hospital pediátrico público de referencia.

\section{MATERIALES Y MÉTODOS}

Estudio observacional, descriptivo, retrospectivo con componente analítico. Se revisaron las historias clínicas de pacientes pediátricos de 0 a 18 años de edad atendidos en el Consultorio de Oftalmología del Hospital Pediátrico Niños de Acosta Ñu de San Lorenzo, Paraguay en el período comprendido entre el 1 de enero de 2011 al 31 de mayo de 2014. Se incluyeron pacientes con TO, cuyo diagnostico se realizo por la presencia de lesiones de corioretinitis, con lesiones activas e inflamatorias, o inactivas, con localización central o periférica. La toxoplasmosis fue considerada congénita por serología materna y del lactante. Se excluyeron los pacientes con fichas clínicas incompletas. Las variables estudiadas fueron: edad, sexo, forma de toxoplasmosis (adquirida o congénita) tipo de lesión ocular en el fondo de ojo, el estado de las lesiones (activas o inactivas), afectación del haz maculo papilar (central o periférica), compromiso ocular uni o bilateral y presencia de complicaciones (estrabismo, desprendimiento de retina y catarata). Los datos fueron analizando con SPSS v 21. Las variables cuantitativas de distribución no normal se expresaron en mediana con rangos intercuartílicos, las cualitativas en porcentajes y la asociación de las mismas utilizando la prueba de chi cuadrado y el test de Fisher según correspondía. Se consideró un error alfa menor a 5\%. El protocolo fue aprobado por el comité de ética de la investigación del hospital, y no se requirió de consentimiento informado.

\section{RESULTADOS}

En el periodo de estudio 9554 pacientes fueron atendidos en el consultorio de oftalmología pediátrica. Llenaron los criterios de inclusión 72 pacientes (0,8\%). El 43\% (31/72) fue remitido desde 
otro servicio del hospital, para evaluación oftalmológica por diagnostico de toxoplasmosis y el $57 \%(41 / 72)$ fue de pacientes que acudieron por diversas motivos y que presentaron lesiones oculares compatibles de TO. La mediana de edad fue de 48 meses con rango intercuartilico de 14(P25) y 141(P75). La edad clasificada en grupos etarios, así como el sexo se observa en la Tabla 1.

Tabla 1. Características demográficas de los pacientes pediátricos con Toxoplasmosis ocular. $\mathrm{N}=72$.

\begin{tabular}{llll}
\hline & & N & $(\%)$ \\
\hline Grupo etario & Neonatos & 5 & $(6,9)$ \\
& Lactantes & 19 & $(26,4)$ \\
& 25 m a 9 años & 26 & $(36,1)$ \\
Sexo & 10 a 18 años & 22 & $(30,6)$ \\
& Femenino & 38 & $(52.8)$ \\
\hline
\end{tabular}

El 80,5\%(58/72) fueron toxoplasmosis adquirida (TA) y el 19,4\%(14/72) congénitas (TC).

Todos los pacientes tenían coriorretinitis. En el 7\% (5/72) se acompañaron de uveítis. La afectación fue bilateral en $52.8 \%(38 / 72)$. Las lesiones eran inactivas en $87,5 \%(63 / 72)$ y activas en $12,5 \%$ (9/72). La frecuencia de estas lesiones según los grupos de edad, en la TC y la TA se observa en la Tabla 2.

Tabla 2. Grupo etario y actividad de las lesiones en la toxoplasmosis congénita y adquirida con lesiones oculares. $\mathrm{N}=72$.

\begin{tabular}{lccc}
\hline Tipo de lesiones & $\begin{array}{c}\text { Toxoplasmosis } \\
\text { Adquirida } \\
\mathrm{N}(\%)\end{array}$ & $\begin{array}{c}\text { Toxoplasmosis } \\
\text { Congénita } \\
\mathrm{N}(\%)\end{array}$ & $\mathbf{p}$ \\
\hline Activas & $7(12)$ & $2(14)$ & $0.55^{*}$ \\
Inactivas & $51(88)$ & $12(86)$ & \\
Grupo etario & & & \\
$\begin{array}{l}\text { Neonatos y lactantes menores } \\
\text { Mayores de 24 meses }\end{array}$ & $13(22)$ & $10(71)$ & $0.001^{*}$ \\
\hline * test de Fisher & $45(78)$ & $4(29)$ & \\
\hline
\end{tabular}

Analizando las corioretinitis en lo referente a su localización, se encontró que el 69,4\%(50/72) eran centrales, es decir afectaban el haz papilomacular, el $5,6 \%(4 / 72)$ eran periféricas y en el $25 \%(18 / 72)$ eran mixtas.

Presentaron complicaciones atribuibles a las lesiones causadas por toxoplasmosis en el 58,3\% (42/72) de los pacientes. La distribución de complicaciones según edad se observa en la Tabla 3.
Tabla 3. Frecuencia de complicaciones de acuerdo a los grupos etarios considerados en pacientes con lesiones oculares por toxoplasmosis congénita. $\mathrm{n}=42$.

\begin{tabular}{llcc}
\hline \multirow{2}{*}{ Grupo etario } & Complicaciones & \\
\hline \multicolumn{2}{c}{} & N (\%) & p \\
\hline & Neonatos y lactantes & $17(40)$ & \\
& $>24$ meses & $25(60)$ & $0.20 *$ \\
\hline
\end{tabular}

Los tipos de complicaciones encontradas fueron: estrabismo en $76 \%$ (32/42), desprendimiento de retina en $24 \%(10 / 42)$.

\section{DISCUSIÓN}

En el presente estudio la frecuencia de $\mathrm{TO}$ en el consultorio de oftalmología pediátrica fue baja, comparando con reportes de otros países. En México, un estudio realizado en pacientes pediátricos de una clínica oftalmológica, encontraron una prevalencia de $11,2 \%$ de $\mathrm{TO}^{(1)}$. En Latinoamérica, Arantes y cols en un estudio de cohortes retrospectivo realizado en el sur del Brasil, encontraron una incidencia de 6,8/100 pacientes año de toxoplasmosis ocular en una población de niños y adultos con toxoplasmosis adquirida. Esta región tiene una elevada prevalencia de toxoplasmosis ${ }^{(6)}$. En la provincia argentina de Misiones se ha reportado toxoplasmosis ocular en el $20 \%$ de los pacientes adultos de un consultorio oftalmológico $^{(7)}$. En África, en la región central de Ghana, se encontró una prevalencia de $2,6 \%{ }^{(8)}$. En Malasia, en una clínica oftalmológica general, en un periodo de 6 años (2010 al 2016) detectaron solo 16 casos de $\mathrm{TO}^{(9)}$. La variabilidad también se observa en distintas regiones de un mismo país como es el caso de Brasil, donde en un estudio realizado en banco de ojos, se encontró TO en el $7 \%$ de los ojos de las personas provenientes de Sao Paulo frente al $87 \%$ de los provenientes de Joinville.(5) Las variaciones observadas tanto en la frecuencia como en la severidad de las lesiones oculares, podrían deberse a factores dependientes del parásito tales como la diversidad genética del T. gondii y la emergencia de especies atípicas. Se relacionan además con factores dependientes del huésped, como las características y tipos de respuesta inmune ${ }^{(10)}$. Un estudio comparativo de TO en colombianos y franceses, demostró las diferencias tanto en gravedad como en la evolución de la TO. En los primeros predominó el 
genotipo I del parasito y algunas cepas atípicas, así como niveles elevados de interleukinas 3 y 6 (IL-3 y IL6) mientras que en la población francesa predomino el tipo II. Los elevados niveles de estas interleukinas observadas en la población colombiana podría estaría asociados a las formas graves de TO en Sudamérica ${ }^{(11)}$.

La mayoría de los pacientes del presente estudio tenían toxoplasmosis adquirida. En Brasil se ha encontrado elevada frecuencia de $\mathrm{TO}$ en la toxoplasmosis adquirida y constituyó un importante hallazgo, al punto de ser comentado en la editorial de una importante revista como el primer punto de las lecciones aprendidas del Brasil sobre esta infección ${ }^{(6,12)}$.

Una tercera parte de la población estudiada era lactante y en este grupo se detectaron los casos de TC. La lesión ocular en esta infección congénita puede verse hasta en un $70 \%$ de los casos y frecuentemente asociada a otras afectaciones neurológicas ${ }^{(13)}$. La mayoría de los pacientes con TO, aun aquellos con TC, tenían lesiones inactivas, es decir, fueron cicatrízales. Melamed y cols encontraron $84 \%$ de lesiones inactivas en la TC en una región del Brasil, cifras muy similares a la encontrada en el presente estudio.(13) Estos hallazgos se relacionan con la consulta tardía y por tanto, los expone a tener alteraciones visuales severas, con la consiguiente disminución de la calidad de vida de los afectados ${ }^{(14,15)}$.

Arantes y cols encontraron que el tratamiento precoz y adecuado de la toxoplasmosis se asoció a menor afectación ocular ${ }^{(6)}$. Sin embargo, existe aun controversia en relación a la combinación de varias drogas, el uso de tratamiento sistémico versus intravítreo y la utilización de esteroides. De acuerdo con la última revisión Cochrane, el tratamiento de la corioretinitis por toxoplasmosis podría disminuir el riesgo de recurrencia ${ }^{(3,16-18)}$. Especial cuidado debe tenerse en pacientes inmunocomprometidos, con lesiones activas de la cámara posterior, considerando que pueden presentar formas fulminantes de $\mathrm{TO}^{(19)}$. Los pacientes del presente estudio recibieron tratamiento indicado en forma conjunta con el servicio de infectología del hospital.

El estudio de Arantes y cols demostró la importancia del seguimiento de los pacientes considerados con riesgo de TO. Muchos pacientes del estudio que no tenia lesiones oculares en la primera evaluación, lo presentaron en el seguimiento con una incidencia de corioretinitis necrótica de 6,5/100 pacientes/año ${ }^{(6)}$. Además las lesiones en la TO pueden reactivarse, o presentar recurrencia de la infección ${ }^{(20)}$. Recientemente se ha identificado una proteína sérica, la peptidil prolys cis trans isomerasa A (PPIA por sus siglas en inglés), como un biomarcador de recurrencia en la $\mathrm{TO}^{(21)}$.

En este estudio todos los pacientes tenían corioretinitis, acompañado en menor porcentaje de uveítis. De hecho, la toxoplasmosis es considerada como la primera causa de lesiones de la cámara posterior del ojo, tal como lo reportan varios estudios $^{(6-8)}$. En Japón la toxoplasmosis es una de las principales causa de uveítis ${ }^{(22)}$.

La fisiopatología de la lesión ocular posiblemente se deba a la acción del T.gondii sobre los macrófagos y las células dendríticas de la retina, quienes liberan citoquinas inflamatorias principalmente interleukinas. Sin embargo, la capacidad de producir lesiones se relacionan con polimorfismos genéticos del parasito y los mediadores inflamatorios del huésped $^{(14,23)}$.

En más de la mitad de los casos de TO, la afectación fue bilateral como en muchos estudios ${ }^{(2,13)}$. La afectación unilateral es más frecuente en adultos. En lo referente a las complicaciones, encontradas, estas fueron elevadas, posiblemente relacionadas con el diagnostico tardío de la TO.

El presente estudio tiene las limitaciones de ser un estudio retrospectivo, con un escaso número de pacientes. El diagnóstico de toxoplasmosis adquirida se realizó, en algunos pacientes, por criterios clínicos porque no se disponía de resultados de serología. Sin embargo, la información proporcionada, puede constituir un punto de partida para un estudio prospectivo que pueda proporcionar información sobre la frecuencia y la valoración de la toxoplasmosis como un problema serio de salud pública, teniendo en cuenta la gravedad de las lesiones oculares que produce. Se debe considerar además las diferencias regionales 
de esta infección, y la cercanía geográfica del país, a regiones del Brasil, con elevada prevalencia de Toxoplasmosis.

\section{CONCLUSIONES}

La frecuencia de $\mathrm{TO}$ ocular en el consultorio de oftalmología pediátrica fue del 0,8\%. La mayoría de los pacientes tenían más de 2 años de edad, de los cuales poco menos de la mitad era adolescente. El

\section{REFERENCIAS BIBLIOGRÁFICAS}

1. Garza-Leon M, Arellanes Garcia L. Ocular toxoplasmosis: Clinical characteristics in pediatric patients. Ocul Immunol Inflamm. 2012; 20(2):130-8.

2. Khan K, Khan W. Congenital toxoplasmosis: An overview of the neurological and ocular manifestations. Parasitol Int. 2018; 67(6):715-21. Doi: https://doi.org/ 10.1016/j.parint.2018.07.004

3. Cerqueira Lima GS, Saraiva PGC, Saraiva FP. Current Therapy of Acquired Ocular Toxoplasmosis: A Review. J Ocul Pharmacol Ther. 2015; 31(9):511-7. Doi: http://online.liebertpub.com/doi/10.1089/jop.2015.0059

4. Furtado JM, Winthrop KL, Butler NJ, Smith JR. Ocular toxoplasmosis I: parasitology, epidemiology and public health. Clin Experiment Ophthalmol. 2013; 41(1):82-94. Doi: http://doi.wiley.com/10.1111/j.14429071.2012.02821.x

5. Commodaro Alessandra,Chiasson Melissa, Sundar Natarajan,Rizzo Luiz Vicente,Belfort Rubens GME. Elevated toxoplasma gondii infection rates for retinas from eyes bank southern Brazil. Emerg Infect Dis. 2015; 21(4):691-3.

6. Arantes TEF, Silveira C, Holland GN, Muccioli C, Yu F, Jones JL, et al. Ocular involvement following postnatally acquired toxoplasma gondii infection in southern brazil: A 28-year experience. Am J Ophthalmol. 2015; 159(6):1002-1012.e2.

7. Rudzinski M, Meyer A. Prevalencia y factores de riesgo asociados con toxoplasmosis ocular en la zona centroeste de la provincia de Misiones, Argentina. Oftalmol Clin Exp. 2011;4(4):159-62.

8. Abu EK, Boampong JN, Amoabeng JK, Ilechie AA, Kyei S, Owusu-Ansah A, et al. Epidemiology of Ocular
19\% tenía toxoplasmosis congénita. La afectación bilateral fue la más frecuente.

Todos presentaron corioretinitis, en la mayoría de los casos centrales y con lesiones inactivas, tanto la toxoplasmosis congénita como la adquirida. Más de la mitad de los pacientes presentaron complicaciones en el momento del diagnostico, principalmente el grupo de niños mayor de 2 años de edad.

Toxoplasmosis in Three Community Surveys in the Central Region of Ghana, West Africa. Ophthalmic Epidemiol. 2016; 23(1):14-9.

9. Jong T, Matthew H, A EZ, B AH, Tajudin A. Clinical profile of ocular toxoplasmosis in the Universiti Sains Malaysia Hospital - 7-year review. Family Medicine \& Primary Care Review 2018; 20(1):13-6.

10. Fernández C, Jaimes J, Ortiz MC, Ramírez JD. Host and Toxoplasma gondii genetic and non-genetic factors influencing the development of ocular toxoplasmosis: A systematic review. Infect Genet Evol. 2016;44:199-209. Doi:http://dx.doi.org/10.1016/j.meegid.2016.06.053

11. de-la-Torre A, Sauer A, Pfaff AW, Bourcier T, Brunet J, Speeg-Schatz C, et al. Severe South American Ocular Toxoplasmosis Is Associated with Decreased Ifn- $\gamma / \mathrm{Il}-17 \mathrm{a}$ and Increased Il-6/Il-13 Intraocular Levels. PLoS Negl Trop Dis. 2013; 7(11).

12. Grigg, Michael E.Dubey Jitender P NRB. Ocular Toxoplasmosis:lessons From Brazil. Am J Ophthalmol. 2015; 159(6):999-1001.

13. Melamed J, Eckert GU, Spadoni VS, Lago EG, Uberti F. Ocular manifestations of congenital toxoplasmosis. Eye. 2010; 24(4):528-34.

14. Pleyer U, Schlüter D, Mänz M. Ocular toxoplasmosis: Recent aspects of pathophysiology and clinical implications. Ophthalmic Res. 2014;52(3):116-23.

15. De-La-Torre A, González-López G, MontoyaGutiérrez JM, Marín-Arango V, Gómez-Marín JE. Quality of life assessment in ocular toxoplasmosis in a Colombian Population. Ocul Immunol Inflamm. 2011; 19(4):262-6.

16. De-La-Torre A, Stanford M, Curi A, Jaffe GJ, Gomez- 
Marin JE. Therapy for ocular toxoplasmosis. Ocul Immunol Inflamm. 2011;19(5):314-20.

17. Harrell M, Carvounis PE. Current treatment of toxoplasma retinochoroiditis: An evidence-based review. JOphthalmol. 2014;2014.

18. Pradhan E, Bhandari S, Gilbert RE, Stanford M. Antibiotics versus no treatment for toxoplasma retinochoroiditis. Cochrane Database Syst Rev. 2016; 2016(5).

19. Oray M, Ozdal PC, Cebeci Z, Kir N, Tugal-Tutkun I. Fulminant Ocular Toxoplasmosis: The Hazards of Corticosteroid Monotherapy. Ocul Immunol Inflamm. 2016; 24(6):637-46.

20. Persons N, Province M, Rudzinski M, Khoury M, Couto C, Ajzenberg D. Reactivation of Ocular
Toxoplasmosis in Tropical Islands as New Hubs for Emerging Arboviruses. 2016;22(5):912-3.

21. Isenberg J, Golizeh M, Belfort RN, da Silva AJ, Burnier NM, Ndao M. Peptidyl-prolyl cis-trans isomerase A - A nivel biomarker of multi-episodic (recurrent) ocular oxoplasmosis. Exp Eye Res. 2018; 177:104-11. Doi: https://doi.org/10.1016/j.exer.2018.07.029

22. Takeda A, Ishibashi T, Sonoda KH. Epidemiology of Uveitis, Caused by HTLV-1, Toxoplasmosis, and Tuberculosis; the Three Leading Causes of Endemic Infectious Uveitis in Japan. Ocul Immunol Inflamm. 2017; 25(Supp 1):S19-23.

23. Kijlstra A, Petersen E. Epidemiology, pathophysiology, and the future of ocular toxoplasmosis. Ocul Immunol Inflamm. 2014; 22(2):138-47. 